\title{
GENERAL CONTACT-ANGLE CONDITIONS WITH AND WITHOUT KINETICS
}

\author{
BY \\ SIGURD ANGENENT (Department of Mathematics, University of Wisconsin, Madison, WI) \\ AND
}

MORTON E. GURTIN (Department of Mathematics, Carnegie Mellon University, Pittsburgh, PA)

\begin{abstract}
The classical condition for the contact angle of a phase interface at a container wall is generalized to include both anisotropy and kinetics. The derivation, which does not involve an assumption of local equilibrium, is based on a capillary force balance, a dissipation inequality representing the second law, and suitable constitutive assumptions.
\end{abstract}

1. Introduction. Consider a vessel containing phases $\alpha$ and $\beta$ separated by a sharp interface. The classical condition for the contact angle $\gamma$ of the interface at the container wall is

$$
\cos \gamma=g / f, \quad g=g_{\alpha}-g_{\beta},
$$

where $f$ and $g_{p}(p=\alpha, \beta)$ are constants with $f$ the free energy of the interface and $g_{p}$ the free energy of the contact layer between phase $p$ and the container wall. The condition (1.1) is generally derived from a condition of local equilibrium, and it seems reasonable to inquire whether there is a dynamical generalization that accounts for the motion of the contact point along the container wall.

Our main result is a general contact condition that allows for both anisotropy and kinetics:

$$
\mathbf{C}(\theta) \cdot \mathbf{T}(\omega)=g(\omega)+\mu(\theta, \omega, v) v
$$

with $\mu(\theta, \omega, v) \geq 0$ a kinetic modulus and

$$
\mathbf{C}(\theta)=f(\theta) \mathbf{T}(\theta)+f^{\prime}(\theta) \mathbf{N}(\theta)
$$

the capillary force. Here $v$ is the velocity of the contact point along the container wall, $\theta$ is the normal angle of the interface, $\omega$ is the normal angle of the container wall, $\mathbf{T}(\theta)$ and $\mathbf{N}(\theta)$ are the unit tangent and normal to the interface, and $\mathbf{T}(\omega)$ is the unit tangent to the wall. (Although our derivation is in $\mathbb{R}^{2}$, the results apply also to $\mathbb{R}^{3}$, the relevant plane being that spanned by the normal to the interface and the normal to the wall. Precise definitions specifying orientations, etc. are given in the text.)

Received January 25, 1994.

1991 Mathematics Subject Classification. Primary 80A22. 
If $\mu$ is independent of $v$, then, granted isotropy, we are led to a dynamical generalization of $(1.1)$ :

$$
f \cos \gamma=g+\mu v, \quad \cos \gamma=\mathbf{T}(\theta) \cdot \mathbf{T}(\omega)
$$

interestingly, (1.4) places a limitation on the magnitude of the velocity $v$.

We show that - granted certain assumptions - the contact condition (1.2) has two roots

$$
\theta=\Theta^{ \pm}(\omega, v), \quad \omega-\pi<\Theta^{-}(\omega, v)<\omega<\Theta^{+}(\omega, v)<\omega+\pi
$$

For $\mu$ independent of $\theta$ these roots can be obtained graphically using a procedure that is most easily explained for the special case: $\mu=0, g(\omega)>0$. Plot the Frank diagrams $\mathscr{F}$ and $\mathscr{G}$ of $f$ and $g$ (F्F, for example, is the graph, in polar coordinates, of $\left.r=f(\theta)^{-1}\right)$. Assume that $\mathscr{F}$ is convex and enclosed by $\mathscr{G}$ (the "wetting condition"). Then, given any angle $\omega$, consider the point $\mathbf{x}$ on $\mathscr{G}$ that corresponds to the angle $\omega$. There are exactly two lines through $\mathrm{x}$ that are tangent to $\mathscr{F}$; the angles $\theta$ corresponding to these points of tangency are the roots $\theta=\Theta^{ \pm}(\omega)$ of $(1.2)$.

Our derivation of the general condition (1.2) is dynamical from the outset; it is based on a capillary force balance for the contact point, a dissipation inequality representing the second law for the restricted situation under consideration, and suitable constitutive assumptions.

2. Balance of forces. We consider the motion of a two-phase system in a fixed region $\Omega$ in $\mathbb{R}^{2}$. We assume that the phases, labelled $\alpha$ and $\beta$, are separated by a sharp interface at each time. Our interest is in the behavior of the system near a given contact point $\mathbf{z}(t)$ of the interface with the container wall adjacent to $\partial \Omega$. With this in mind, we choose an arbitrary time $t_{0}$ and write

$$
\mathscr{D}(r)=\Omega \cap\left\{\text { open disc of radius } r \text { centered at } \mathbf{z}\left(t_{0}\right)\right\} \text {. }
$$

We assume there is a sufficiently small $r_{0}$ such that, for all $t$ in a sufficiently small neighborhood $\mathscr{T}$ of $t_{0}, \mathbf{z}(t)$ is the only contact point in $\mathscr{D}_{0}=\mathscr{D}\left(r_{0}\right)$.

We henceforth confine attention to the time interval $\mathscr{T}$ and to behavior within $\mathscr{D}_{0}$.

We assume that the portion $s(t)$ of the interface in $\mathscr{D}_{0}$ is a smoothly evolving curve with a time-dependent parametrization whose arc length $s$ increases away from $\mathbf{z}(t)$. In addition, we choose a unit normal $\mathbf{N}(\mathbf{x}, t)$ and unit tangent $\mathbf{T}(\mathbf{x}, t)$ on $s(t)$ oriented as in Fig. 1.

We allow for boundary layers (of zero thickness) between the container wall and each of the phases. We write $b_{p}(t), p=\alpha, \beta$, for the portion of $\partial \Omega \cap \mathscr{D}_{0}$ contained in phase $p$, and we identify these boundary layers with $b_{\alpha}(t)$ and $b_{\beta}(t)$. We assume that $\partial \Omega \cap \mathscr{D}_{0}$ is smooth, we choose a parametrization in which arc length $\sigma$ increases as $\mathbf{z}(t)$ is crossed from $\alpha$ to $\beta$, and we choose a unit normal $\nu(\mathbf{x})$ and unit tangent $\tau(\mathbf{x})$ to $\partial \Omega \cap \mathscr{D}_{0}$ as in Fig. 1. 


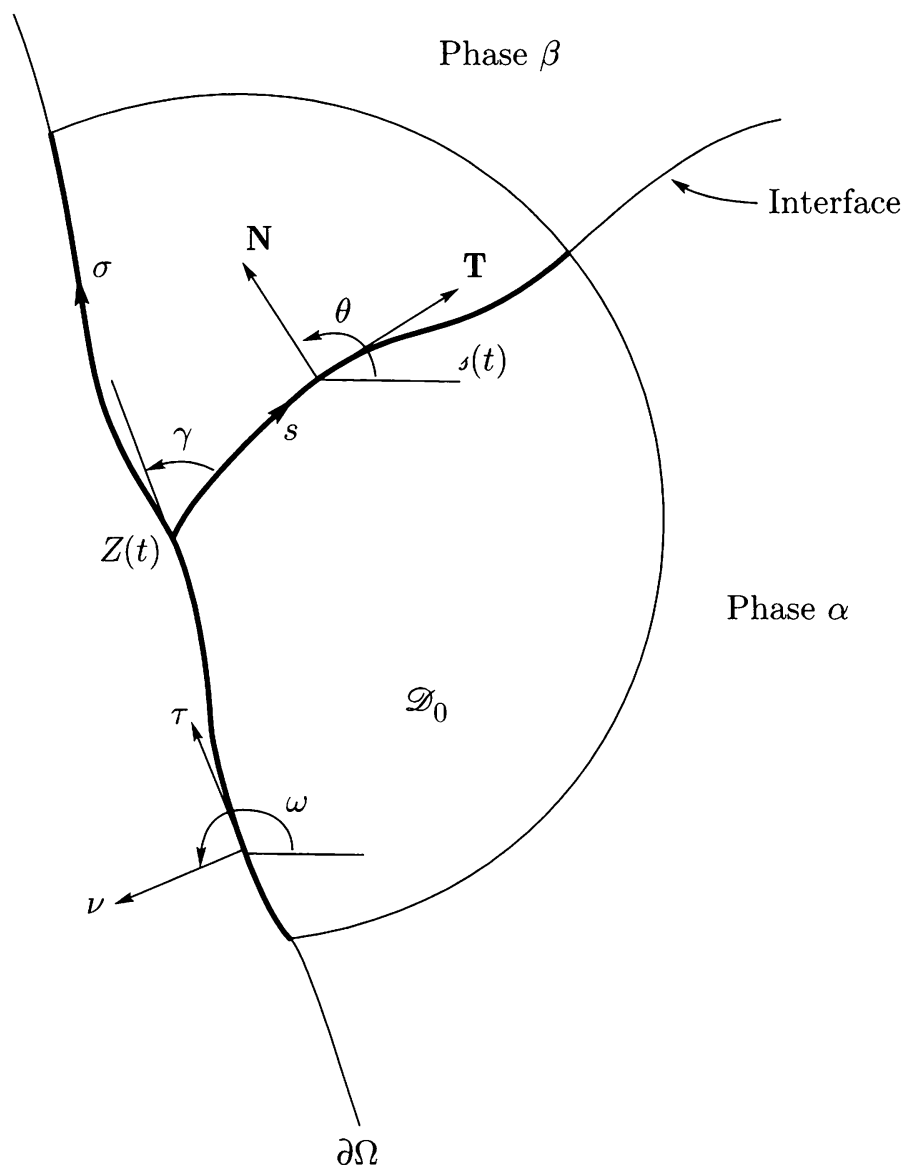

FIG. 1. The region $\mathscr{D}_{0}$ of interest. Sign conventions

Behavior within the interface is described by a capillary force $\mathbf{C}(\mathbf{x}, t)$ on $s(t)$ whose tangential and normal components represent surface tension and surface shear [AG, G1G3]. Let $\mathscr{R}$ with initial and terminal points $\mathbf{x}_{1}$ and $\mathbf{x}_{2}$ be a subcurve of $s(t)$. Then $-\mathbf{C}\left(\mathbf{x}_{1}, t\right)$ and $\mathbf{C}\left(\mathbf{x}_{2}, t\right)$ are the capillary forces exerted on $\mathscr{R}$ across $\partial \mathscr{R}$ at $\mathbf{x}_{1}$ and $\mathbf{x}_{2}$.

We also allow for capillarity at the container walls described by forces $G_{\alpha}(\mathbf{x}, t)$ and $G_{\beta}(\mathbf{x}, t)$ on $b_{\alpha}(t)$ and $b_{\beta}(t)$, respectively. If $\mathscr{B}$-with initial and terminal points $\mathbf{x}_{1}$ and $\mathbf{x}_{2}$-is a subcurve of $b_{\alpha}(t)$, then $-\mathbf{G}_{\alpha}\left(\mathbf{x}_{1}, t\right)$ and $\mathbf{G}_{\alpha}\left(\mathbf{x}_{2}, t\right)$ are the capillary forces exerted on $\mathscr{B}$ across $\partial \mathscr{B}$ at $\mathbf{x}_{1}$ and $\mathbf{x}_{2}$, and similarly for $b_{\beta}(t)$.

Finally, we associate with $\mathbf{z}(t)$ a concentrated force $\mathbf{P}(t)$ acting at $\mathbf{z}(t) ; \mathbf{P}(t)$ represents the force exerted by the container at the junction (of the interface and the boundary layers).

Consider an arbitrary "control volume" $\mathscr{D}(r)\left(r<r_{0}\right)$. Let

$$
s(r, t)=s(t) \cap \mathscr{D}(r), \quad b_{p}(r, t)=b_{p}(t) \cap \mathscr{D}(r),
$$

and let $\mathbf{z}(t)$ and $\mathbf{y}(r, t)$ denote the initial and terminal points of $s(r, t) ; \mathbf{x}_{1}(r)$ and $\mathbf{z}(t)$ the initial and terminal points of $b_{\alpha}(r, t) ; \mathbf{z}(t)$ and $\mathbf{x}_{2}(r)$ the initial and terminal points 


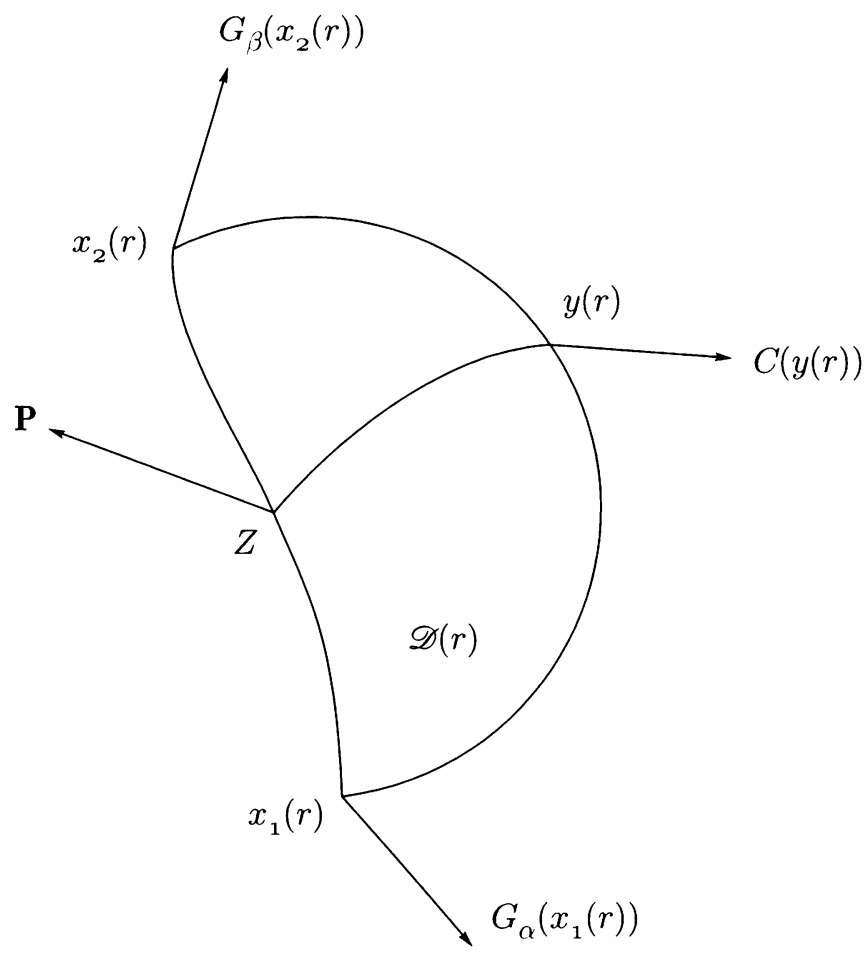

FIG. 2. Forces on a control volume $\mathscr{D}_{0}(r)$

of $b_{\beta}(r, t)$. Then, suppressing the argument $t$, we consider balance of forces for $\mathscr{D}(r)$ in the form (Fig. 2)

$$
\mathbf{C}(\mathbf{y}(r))+\mathbf{G}_{\beta}\left(\mathbf{x}_{2}(r)\right)-\mathbf{G}_{\alpha}\left(\mathbf{x}_{1}(r)\right)+\mathbf{P}+o(1)=\mathbf{0}
$$

as $r \rightarrow 0$, and therefore arrive at the following force balance for the contact point:

$$
\mathbf{C}-\mathbf{G}+\mathbf{P}=\mathbf{0} \quad \text { at }(\mathbf{z}(t), t)
$$

where

$$
\mathbf{G}(t)=\mathbf{G}_{\alpha}(\mathbf{z}(t), t)-\mathbf{G}_{\beta}(\mathbf{z}(t), t),
$$

and where, for example, $\mathbf{G}_{\beta}(\mathbf{z}(t), t)$ represents the limit of $\mathbf{G}_{\beta}(\mathbf{x}, t)$ as $\mathbf{x} \rightarrow \mathbf{z}(t)$ from $b_{\beta}(t)$. The term of $o(1)$ represents continuously distributed forces whose net contribution vanishes as $r \rightarrow 0$.

\section{Dissipation inequality.}

a. Free energies. We write $f(\mathbf{x}, t)>0$ for the free energy, per unit length, of the interface $s(t)$, and, for $p=\alpha, \beta$, we let $g_{p}(\mathbf{x}, t) \geq 0$ denote the free energy, per unit length, of the boundary layer $b_{p}(t)$; then, granted the equivalence of surface tension and free energy,

$$
f=\mathbf{C} \cdot \mathbf{T}, \quad g_{p}=\mathbf{G}_{p} \cdot \tau
$$


The total free energy $\mathscr{E}(r, t)$ of the interface and boundary layer in a "control volume" $\mathscr{D}(r)$ is given by

$$
\mathscr{E}(r, t)=\int_{\jmath(r, t)} f d s+\int_{\mathscr{C}_{\alpha}(r, t)} g_{\alpha} d \sigma+\int_{\mathscr{\ell}_{\beta}(r, t)} g_{\beta} d \sigma,
$$

where here and in what follows we use the notation in the paragraph containing (2.2). Thus, suppressing the argument $t$,

$$
\left\{\int_{s(r)} f d s\right\}^{\bullet}=f(\mathbf{y}(r)) \mathbf{y}^{\cdot}(r) \cdot \mathbf{T}(\mathbf{y}(r))-f(\mathbf{z}) \mathbf{z} \cdot \mathbf{T}(\mathbf{z})+\int_{s(r)}(\ldots) d s
$$

where the integrand $(\ldots)$ is unimportant, and where a superscript dot denotes differentiation with respect to $t$ (cf. [G2], Eq. (2.24)). Similar expressions apply to the other two integrals in (3.2); these and (3.3) imply that, as $r \rightarrow 0$,

$$
\mathscr{E} \cdot(r)=f(\mathbf{z})\left[\mathbf{y}^{\cdot}(0) \cdot \mathbf{T}(\mathbf{z})-\mathbf{z}^{\cdot} \cdot \mathbf{T}(\mathbf{z})\right]+g \mathbf{z}^{\cdot} \cdot \tau(\mathbf{z})+o(1)
$$

where

$$
g(t)=g_{\alpha}(\mathbf{z}(t), t)-g_{\beta}(\mathbf{z}(t), t)
$$

and where $\mathbf{y}^{\cdot}(0)$ denotes the limit of $\mathbf{y}^{\cdot}(r)$ as $r \rightarrow 0$.

The theory under consideration is purely mechanical, with the interface driven by bulk free-energy differences. If $F_{\alpha}$ and $F_{\beta}$, assumed constant, denote the bulk free energies of $\alpha$ and $\beta$, then, letting $F(\mathbf{x}, t)$ denote the piecewise constant function that has the value $F_{\alpha}$ in phase $\alpha$ and $F_{\beta}$ in phase $\beta$, the total bulk energy $E(r, t)$ of $\mathscr{D}(r)$ is given by

$$
E(r, t)=\int_{\mathscr{D}(r)} F(\mathbf{x}, t) d a
$$

The rate $E^{\cdot}(r, t)$ is then the integral over $s(r)$ of $F_{\alpha}-F_{\beta}$ times the normal velocity of $s(r)$, and therefore, as $r \rightarrow 0$,

$$
E^{\cdot}(r, t)=o(1)
$$

b. Expended power. We suppose that the total power expended on $\mathscr{D}(r)$ is given by

$$
\mathscr{P}(r, t)=\mathbf{C}(\mathbf{y}(r, t), t) \cdot \mathbf{y}^{\cdot}(r, t)+o(1)
$$

as $r \rightarrow 0$. The forces $\mathbf{G}_{\alpha}\left(\mathbf{x}_{1}(r), t\right)$ and $\mathbf{G}_{\beta}\left(\mathbf{x}_{2}(r), t\right)$ act on $\partial \mathscr{D}(r)$, but do not perform work, since $\mathbf{x}_{1}(r)$ and $\mathbf{x}_{2}(r)$ do not vary with time; the forces $\mathbf{C}(\mathbf{z}(t), t), \mathbf{G}_{\alpha}(\mathbf{z}(t), t)$, and $\mathbf{G}_{\beta}(\mathbf{z}(t), t)$ do not enter (3.8), since they act internally to $\mathscr{D}(r)$. Finally, the force $\mathbf{P}(t)$ acts on $\partial \mathscr{D}(r)$ at $\mathbf{z}(t)$, and although $\mathbf{P}(t)$ is transported with $\mathbf{z}(t), \mathbf{P}(t)$ performs no work, since the boundary layer itself is stationary. 
c. Dissipation inequality. We base the theory on a dynamical version of the second law, the dissipation inequality, which requires that the energy of a control volume $\mathscr{D}(r)$ change at a rate not greater than the power expended on $\mathscr{D}(r)$ :

$$
\mathscr{E} \cdot(r, t)+E^{\cdot}(r, t) \leq \mathscr{P}(r, t)
$$

Letting $r \rightarrow 0$, we conclude, with the aid of (3.4), (3.7), and (3.8), that

$$
f(\mathbf{z})\left[\mathbf{y}^{\cdot}(0) \cdot \mathbf{T}(\mathbf{z})-\mathbf{z} \cdot \mathbf{T}(\mathbf{z})\right]+g \mathbf{z} \cdot \tau(\mathbf{z}) \leq \mathbf{C}(\mathbf{z}) \cdot \mathbf{y}^{\cdot}(0)
$$

where we have again suppressed the argument $t$.

We denote by $V(t)$ the normal velocity of the interface at the contact point, and by $v(t)$ the velocity of the contact point along the container wall:

$$
V=N(\mathbf{z}) \cdot \mathbf{y}^{\cdot}(0)=\mathbf{N}(\mathbf{z}) \cdot \mathbf{z}^{\cdot}, \quad \mathbf{z}^{\cdot}=v \tau(\mathbf{z})
$$

By (2.5) and (3.5),

$$
g=\mathbf{G}(\mathbf{z}) \cdot \tau(\mathbf{z})
$$

and using (2.4), (3.11), and (3.12), we can reduce the inequality (3.10) to the simple form

$$
P_{\mathrm{tan}} v \leq 0
$$

which asserts that the tangential force

$$
P_{\text {tan }}=\mathbf{P} \cdot \tau
$$

exerted by the container wall on the junction of the interface and the boundary layers dissipates energy over the velocity of the junction along the container wall.

Note that, by (3.12), the tangential part (with respect to $\tau$ ) of the force balance (2.4) yields

$$
\mathbf{C} \cdot \tau-g+P_{\tan }=0
$$

4. Constitutive assumptions. It is convenient to introduce the angle $\theta(t)$ from the (1,0)-axis in $\mathbb{R}^{2}$ to the normal $\mathbf{N}(\mathbf{z}(t), t)$ and the angle $\omega(t)$ from the $(1,0)$-axis to the normal $\nu(\mathbf{z}(t), t)$, with both angles measured counterclockwise. Then $\mathbf{N}(\mathbf{z}(t), t)$ and $\mathbf{T}(\mathbf{z}(t), t)$ may be considered as functions of $\theta(t)$ :

$$
\mathbf{N}(\theta)=(\sin \theta, \cos \theta), \quad \mathbf{T}(\theta)=(-\cos \theta, \sin \theta)
$$

Further, using the functional relations (4.1), we can write $\nu(\mathbf{z}(t), t)$ and $\tau(\mathbf{z}(t), t)$ as

$$
\nu(\mathbf{z}(t), t)=\mathbf{N}(\omega(t)), \quad \tau(\mathbf{z}(t), t)=\mathbf{T}(\omega(t)) .
$$

As constitutive assumptions we assume that the free energy of the interface at the contact point is a function of the normal angle of the interface, and that the difference 
(3.5) in the boundary energies of the two phases at the contact point is a function of the normal angle of the container wall:

$$
f=f(\theta), \quad g=g(\omega) .
$$

In addition, we assume that the free energy $f(\theta)$ generates the capillary force $\mathbf{C}$ through the constitutive equation $[\mathrm{AG}, \mathrm{G} 1, \mathrm{G} 2]$

$$
\mathbf{C}=\mathbf{C}(\theta)=f(\theta) \mathbf{T}(\theta)+f^{\prime}(\theta) \mathbf{N}(\theta) .
$$

Finally, we assume that the tangential force is a function $P_{\tan }=P_{\tan }(\theta, \omega, v)$ of $\theta, \omega$, and the tangential velocity $v$; granted smoothness, the most general such relation of this form consistent with the dissipation inequality (3.13) is

$$
P_{\mathrm{tan}}=-\mu(\theta, \omega, v) v,
$$

with $\mu(\theta, \omega, v) \geq 0$, the kinetic modulus, a constitutive property.

The contact point is constrained to move along the container wall, and the normal force $\mathbf{P} \cdot \mathbf{N}(\omega)$ is a reaction to this constraint; for that reason we do not write a constitutive equation for $\mathbf{P} \cdot \mathbf{N}(\omega)$, but consider it instead as determined by the normal component of the force balance (2.4).

5. Contact-angle conditions. Combining the constitutive equations (4.2)-(4.4) with the force balance (3.15) yields the general contact condition

$$
\mathbf{C}(\theta) \cdot \mathbf{T}(\omega)=g(\omega)+\mu(\theta, \omega, v) v .
$$

In contrast to more classical conditions, (5.1) is dynamical; it furnishes a condition relating the normal angle of the interface, the normal angle of the container wall, and the velocity of the contact point along this wall.

Assume that $\mu$ is independent of $v$; then, granted isotropy, $f, g$, and $\mu$ are constants and

$$
f \mathbf{T}(\theta) \cdot \mathbf{T}(\omega)=g+\mu v ;
$$

thus, defining $\gamma \in[0, \pi]$ as the angle between $\mathbf{T}(\theta)$ and $\mathbf{T}(\omega)$, so that

$$
\cos \gamma=\mathbf{T}(\theta) \cdot \mathbf{T}(\omega), \quad \sin \gamma=\mathbf{N}(\theta) \cdot \mathbf{T}(\omega),
$$

we find that

$$
f \cos \gamma=g+\mu v
$$

which is a dynamical counterpart of the classical condition $f \cos \gamma=g$ for the contact angle $\gamma$. If we assume that $\mu>0$ and $|g|<f$, which is the classical "wetting condition", we arrive at a limiting condition on the velocity:

$$
|v|<(f / \mu)(1+\varepsilon), \quad \varepsilon=|g / f| .
$$

The dynamical condition (5.4) can also be written in terms of the normal velocity $V$. Indeed, (3.11) yields $V=v \mathbf{N}(\theta) \cdot \mathbf{T}(\omega)$, so that

$$
f \cos \gamma=g+\mu V \sin \gamma
$$

Thus, in contrast to $v$, the normal velocity $V$ is not limited in size; in fact, for $g=0$,

$$
V=(f / \mu) \cot \gamma,
$$

so that $V$ goes from $+\infty$ to $-\infty$ as $\gamma$ goes from 0 to $\pi$. 


\section{Solution of the general contact condition.}

a. Solution without kinetics. Here we restrict attention to the equilibrium condition

$$
\mathbf{C}(\theta) \cdot \mathbf{T}(\omega)=g(\omega)
$$

If $g(\omega)=0$, then (5.3) and (6.1) yield

$$
\tan \gamma=-f^{\prime}(\theta) / f(\theta),
$$

which results in the classical condition $\gamma=\pi / 2$ when $f$ is constant. We henceforth assume that

$$
g(\omega) \text { never vanishes. }
$$

To find solutions $\theta=\Theta(\omega)$ of the contact condition (6.1), we introduce the Frank diagram $\mathscr{F}$ of the interfacial energy $f ; \mathscr{F}$ is the graph, in polar coordinates $(r, \theta)$, of $r=f(\theta)^{-1}$. Any point $\mathbf{y}$ on $\mathscr{F}$ thus has the form

$$
\mathbf{y}=\mathbf{N}(\theta) / f(\theta),
$$

and, by (4.4),

$$
f(\theta)^{2} \mathbf{C}(\theta)=-(d / d \theta)\{\mathbf{N}(\theta) / f(\theta)\} ;
$$

hence, $\mathbf{C}(\theta)$ has the same direction as the tangent line $\ell$ to $\mathscr{F}$ at the point (6.4). A short computation shows that $\ell$ is the set of points $\mathbf{x}$ that satisfy

$$
\mathbf{x} \cdot \mathbf{Q C}(\theta)=1,
$$

where $\mathbf{Q}$ is the rotation with $\mathbf{Q T}=\mathbf{N}$. Our final step is to rewrite (6.1) as

$$
\Phi(\theta, \omega):=\mathbf{Q C}(\theta) \cdot\{\mathbf{N}(\omega) / g(\omega)\}=1,
$$

which asserts that the point $\mathbf{N}(\omega) / g(\omega)$ lies on the tangent $\ell$ to the Frank diagram $\mathscr{F}$ at the angle $\theta$ (Fig. 3). Thus, if $\omega$ is given, we can find all roots $\theta=\Theta(\omega)$ of $(6.1)$ by drawing all lines through $\mathbf{N}(\omega) / g(\omega)$; those that are tangent to $\mathscr{F}$ give rise to roots of (6.1), and the angles $\theta$ that correspond to the tangencies are precisely these roots.

In what follows we will assume that the "wetting condition"

$$
\begin{array}{cl}
g(\varphi)<f(\varphi) & \text { for all angles } \varphi \text { if } g>0, \\
-g(\varphi)<f(\varphi+\pi) & \text { for all angles } \varphi \text { if } g<0
\end{array}
$$

is satisfied, so that $\mathbf{N}(\omega) / g(\omega)$ is always exterior to $\mathscr{F}$.

Case 1. The Frank diagram $\mathscr{F}$ is smooth and strictly convex (curvature bounded away from zero). This, the most stable situation, results in a corresponding Wulff shape without corners. In this case, for each $\omega$, there are exactly two tangents to $\mathscr{F}$ that pass through $N(\omega) / g(\omega)$, and we have exactly two roots

$$
\theta=\Theta^{ \pm}(\omega)
$$




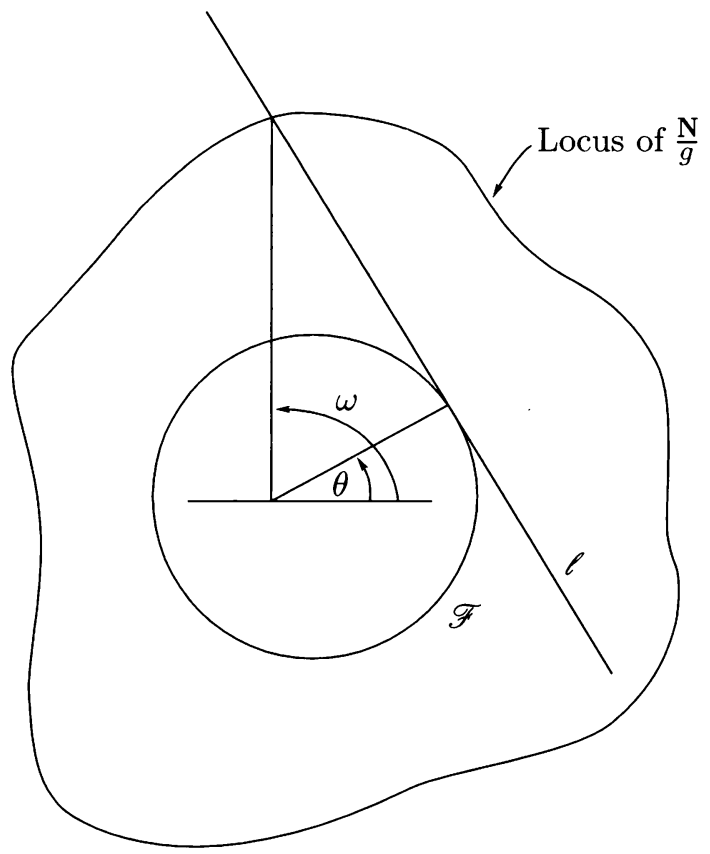

FIG. 3. Graphical solution of the equilibrium condition (6.1) for the case $g>0$

of (6.1). Further, the line through $\mathbf{N}(\omega) / g(\omega)$ and the origin is never tangent to $\mathscr{F}$ and always splits $\mathscr{F}$ into two parts, with each part containing exactly one root. We may therefore order the roots such that

$$
\omega-\pi<\Theta^{-}(\omega)<\omega<\Theta^{+}(\omega)<\omega+\pi .
$$

If $f$ and $g$ are smooth, then the implicit function theorem may be used to show that the roots $\Theta^{ \pm}(\omega)$ of $(6.7)$ are smooth functions of $\omega$. Indeed, it suffices to show that $(\partial / \partial \theta) \Phi(\theta, \omega)$ never vanishes. By $(4.4), \mathbf{C}^{\prime}(\theta)=\left[f(\theta)+f^{\prime \prime}(\theta)\right] \mathbf{N}(\theta)$; hence (6.7) yields

$$
(\partial / \partial \theta) \Phi(\theta, \omega)=-\left\{\left[f(\theta)+f^{\prime \prime}(\theta)\right] / g(\omega)\right\} \mathbf{T}(\theta) \cdot \mathbf{N}(\omega) .
$$

But $\mathbf{T}(\theta) \cdot \mathbf{N}(\omega) \neq 0$ for $\theta=\Theta^{ \pm}(\omega)$, and $f(\theta)+f^{\prime \prime}(\theta) \neq 0$, since the curvature of $\mathscr{F}$ never vanishes (cf. $[\mathrm{AG}]$, Eq. (A5)).

Next,

$$
(\partial / \partial \omega) \Phi(\theta, \omega)=\mathbf{Q} \mathbf{C}(\theta) \cdot(d / d \omega)\{\mathbf{N}(\omega) / g(\omega)\},
$$

and since $\mathbf{Q C}(\theta)$ is normal to $\mathscr{F}$ at $\theta$, while $(d / d \omega)\{\mathbf{N}(\omega) / g(\omega)\}$ is tangent to the locus of $\mathbf{N}(\omega) / g(\omega)$, it follows from the strict convexity of $\mathscr{F}$ that the roots $\Theta^{ \pm}(\omega)$ will be strictly monotone functions of $\omega$ provided the locus of $\mathbf{N}(\omega) / g(\omega)$ is also strictly convex. Figure 4 on p. 566 gives examples in which nonconvexity of this locus yields nonmonotonicity; but there are examples in which it does not.

We now drop the assumptions that $\mathscr{F}$ be convex and smooth. We assume that $f^{\prime}(\theta)$ is piecewise continuous, with jump discontinuities referred to as sharp spots. We let $\mathscr{C}(\mathscr{F})$ 


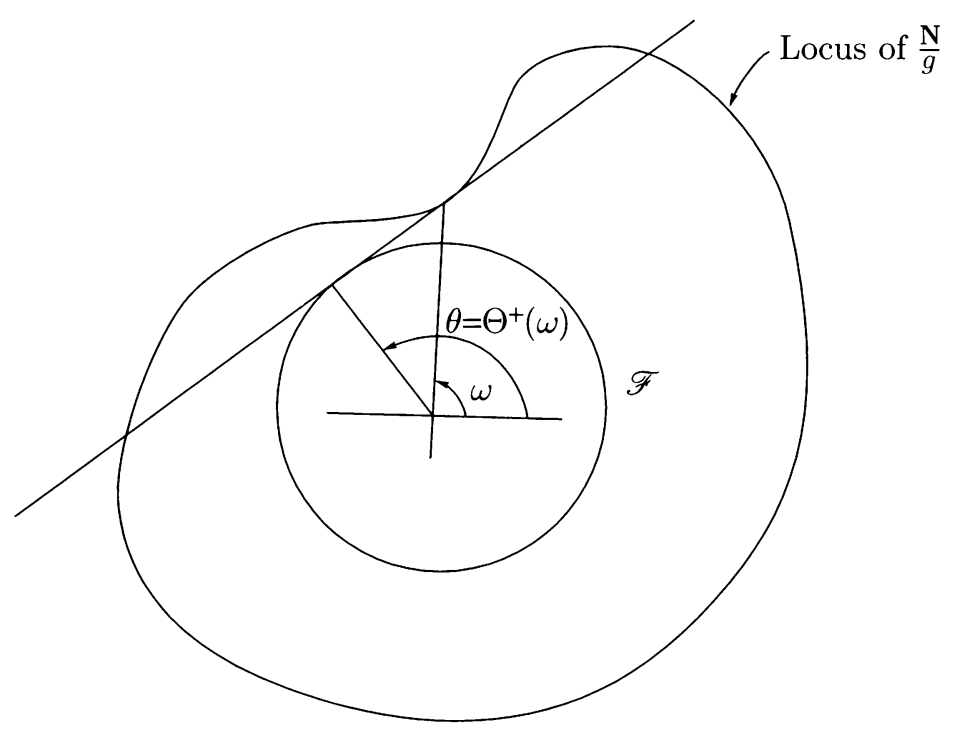

FIG. 4. An example with $\theta=\Theta^{+}(\omega)$ not monotone. It is assumed that $g>0$.

denote the convexification of $\mathscr{F}$ (the boundary of the convex hull of $\mathscr{F}$ ); we refer to angles $\theta$ at which $\mathscr{F}$ and $\mathscr{C}(\mathscr{F})$ coincide as globally stable; and we refer to a tangent $\ell$ to $\mathscr{C}(\mathscr{F})$ as critical if $\ell \cap \mathscr{C}(\mathscr{F})$ is a nontrivial line segment or if $\ell \cap \mathscr{C}(\mathscr{F})$ is a sharp spot $\mathbf{y}$ with $\ell$ tangent to one of the two smooth curves of $\mathscr{C}(\mathscr{F})$ that meet at $\mathbf{y}$. Finally, we refer to $\mathscr{F}$ as regular if [AG, p. 359]:

(i) $\mathscr{F}$ is strictly convex at globally stable angles;

(ii) the set of globally stable angles contains no isolated angles;

(iii) the critical tangents are finite in number, and each such tangent intersects $\mathscr{F}$ at most at two points.

We now look for solutions (6.9) of (6.1) with $\theta$ globally stable. (Such angles represent normal angles at which the interface is, in some sense, stable (cf. [AG, §4.3, §8.1]; [G2, $\S 7])$.)

Case 2. The Frank diagram $\mathscr{F}$ is regular, but not convex and possibly not smooth. As before, for any angle $\omega_{0}$ there are exactly two lines $\ell^{ \pm}\left(\omega_{0}\right)$ that are tangent to $\mathscr{C}(\mathscr{F})$ and pass through $\mathbf{N}\left(\omega_{0}\right) / g\left(\omega_{0}\right)$. Further, the intersections

$$
L^{ \pm}\left(\omega_{0}\right)=\ell^{ \pm}\left(\omega_{0}\right) \cap \mathscr{F}
$$

(with $\mathscr{F}$ ) lie on opposite sides of--and are disjoint from-the line through $\mathbf{N}\left(\omega_{0}\right) / g\left(\omega_{0}\right)$ and the origin; in fact, we may order these sets such that

$$
L^{-}\left(\omega_{0}\right) \subset\left(\omega_{0}-\pi, \omega_{0}\right), \quad L^{+}\left(\omega_{0}\right) \subset\left(\omega_{0}, \omega_{0}+\pi\right) .
$$

Further, by (iii) in the definition of regularity, each of the sets $L^{ \pm}\left(\omega_{0}\right)$ is either a single point or a pair of points.

We will consider only $L^{-}\left(\omega_{0}\right)$; the discussion for $L^{+}\left(\omega_{0}\right)$ is no different. 
Let $L^{-}\left(\omega_{0}\right)$ be a single point and not a sharp spot. Then, for $\omega$ close to $\omega_{0}, \ell^{-}(\omega)$ will also meet $\mathscr{F}$ at a globally stable angle $\theta$ that depends smoothly on $\omega$. Thus, near $\omega_{0}$ we have a smooth functional relation $\theta=\Theta^{-}(\omega)$.

Let $L^{-}\left(\omega_{0}\right)$ be a sharp spot $\mathbf{y}$. If $\ell^{-}\left(\omega_{0}\right)$ is not a critical tangent, then for all $\omega$ near $\omega_{0}, \ell^{-}(\omega)$ will also pass through $\mathbf{y}$, so that we have a functional relation $\theta=\Theta^{-}(\omega)$ near $\omega_{0}$ with $\Theta^{-}(\omega)$ constant. If $\ell^{-}\left(\omega_{0}\right)$ is a critical tangent, then $\omega_{0}$ is the boundary of an interval on which $\Theta^{-}(\omega)$ is constant.

Let $L^{-}\left(\omega_{0}\right)$ consist of two points, with $\theta_{1}$ and $\theta_{2}$ the corresponding angles. Then, near $\omega_{0}$, say for $\omega \in\left(\omega_{0}-\varepsilon, \omega_{0}+\varepsilon\right)$ there is a functional relation $\theta=\Theta^{-}(\omega)$ that is smooth on $\left(\omega_{0}-\varepsilon, \omega_{0}\right)$ and on $\left(\omega_{0}, \omega_{0}+\varepsilon\right)$ with a jump discontinuity from $\theta_{1}$ to $\theta_{2}$ (or vice versa) at $\omega_{0}$. Arguing as above, if $\theta_{1}$ is a sharp spot, and if $\theta_{1}$ is the limiting value of $\Theta^{-}(\omega)$ at $\omega_{0}$ from $\omega<\omega_{0}$, then $\Theta^{-}(\omega)$ is constant for $\omega<\omega_{0}$ sufficiently close to $\omega_{0}$, and similarly if it is the limiting value from $\omega>\omega_{0}$. An analogous assertion applies to $\theta_{2}$.

These results and results analogous to those given in the paragraphs containing (6.11) and (6.12) may be summarized as follows. For a regular Frank diagram the functions $\theta=\Theta^{ \pm}(\omega)$ giving the two roots of the equilibrium condition (6.1) are well defined and smooth except at a finite number of jump discontinuities, and satisfy (6.10). The jump discontinuities arise from tangents $\ell$ to the convexified Frank diagram $\mathscr{C}(\mathscr{F})$ for which $\ell \cap \mathscr{C}(\mathscr{F})$ is a nontrivial line segment. The functions $\Theta^{ \pm}(\omega)$ are constant on certain intervals that come from sharp spots. If the locus of $\mathbf{N}(\omega) / g(\omega)$ is convex, then the functions $\Theta^{ \pm}(\omega)$ are monotone in $\omega$.

b. Solution with kinetics. Consider next the dynamical contact condition (5.1), but, for convenience, with $\mu$ independent of $\theta$ :

$$
\mathbf{C}(\theta) \cdot \mathbf{T}(\omega)=g(\omega)+\mu(\omega, v) v
$$

This yields, in place of (6.7), the condition

$$
\begin{aligned}
\Phi(\theta, \omega, v) & :=\mathbf{Q C}(\theta) \cdot\{\mathbf{N}(\omega) / \hat{g}(\omega)\}=1, \\
\hat{g}(\omega, v) & =g(\omega)+\mu(\omega, v) v .
\end{aligned}
$$

The argument of the previous section with $g(\omega)$ replaced by $\hat{g}(\omega, v)$ then establishesfor $|v|$ sufficiently small that $\hat{g}(\omega, v)$ is consistent with (6.8)-the existence of functions $\theta=\Theta^{ \pm}(\omega, v)$ representing the roots of $(6.15)$. These functions have properties completely analogous to those established in the last section.

If $\mu$ depends also on $\theta$, then we can use the implicit function theorem to solve for functions $\theta=\Theta^{ \pm}(\omega, v)$, but it seems necessary to assume that $|(\partial / \partial \theta) \mu(\theta, \omega, v) v|$ is sufficiently small.

c. Contact conditions in $\mathbb{R}^{3}$ and in the presence of bulk transport. The contact conditions were derived in $\mathbb{R}^{2}$ within a purely mechanical framework, but they are valid almost without change in $\mathbb{R}^{3}$ and in the presence of heat and mass transport in bulk.

When the underlying space is $\mathbb{R}^{3}$, we simply interpret all forces in terms of their projections onto the plane spanned by the normals to the interface and the container wall. In this case, if $r(t)$ represents the contact curve between the interface and the container wall, then $v$ represents the normal velocity of $r(t)$ on $\partial \Omega$. 
We can easily extend the results to situations involving bulk transport; crucial are: (i) for control volumes $\mathscr{D}(r)$, a force balance (2.3) and a dissipation inequality

$$
\mathscr{E} \cdot(r, t) \leq \mathscr{P}(r, t)+o(1)
$$

as $r \rightarrow 0$, where $\mathscr{E}$ and $\mathscr{P}$ have the forms (3.2) and (3.8), and where the $o(1)$ contributions account for bulk transport; (ii) constitutive equations of the form (4.3)-(4.5), but with constitutive functions allowed to depend also on the limiting values of bulk fields at the contact point. For example, the general two-phase Stefan system with heat transport in bulk and with interfacial energy, entropy, and kinetics-as described in $\S \S 15-17$ of [G2] - is consistent with such assumptions. In particular, $f$ and $g$ are then free energies and the constitutive equations (4.3)-(4.5) include dependences on the limiting value of the temperature at the contact point.

7. Evolution problems. A general evolution equation-relating the normal velocity $V$, the curvature $K$ (with $K$ negative when the center of curvature lies in phase $\alpha$ ), and the normal angle $\theta$ at each point of an interface driven by a constant difference $U=F_{\alpha}-F_{\beta}$ in bulk energies-is

$$
b(\theta) V=h(\theta) K-U
$$

[AG, G2], where

$$
h(\theta)=f(\theta)+f^{\prime \prime}(\theta),
$$

while $b(\theta)>0$ is a kinetic modulus. When the interface evolves in a container, this equation is supplemented by the contact condition

$$
\mathbf{C}(\theta) \cdot \mathbf{T}(\omega)=g(\omega)+\mu(\theta, \omega, v) v
$$

at the intersections of the interface with $\partial \Omega$.

In the presence of isotropy with $\mu$ independent of $v$, and for $U=0$, this system reduces to the curve-shortening equation

$$
V=K
$$

$[\mathrm{Br}, \mathrm{Mu}]$ in conjunction with the contact condition

$$
\cos \gamma=g+\mu v
$$

(modulo a suitable scaling).

Acknowledgment. This work was supported by the Army Research Office and the National Science Foundation. The authors acknowledge valuable discussions with P. Voorhees concerning kinetic interface conditions and concerning ideas of S. Davis on this subject. 


\section{REFERENCES}

[AG] S. Angenent and M. E. Gurtin, Multiphase thermomechanics with interfacial structure. 2. Evolution of an isothermal interface, Arch. Rational Mech. Anal. 108, 323-391 (1989)

[Br] K. A. Brakke, The Motion of a Surface by its Mean Curvature, Princeton University Press, 1978

[G1] M. E. Gurtin, Multiphase thermomechanics with interfacial structure. 1. Heat conduction and the capillary balance law, Arch. Rational Mech. Anal. 104, 185-221 (1988)

[G2] M. E. Gurtin, Thermomechanics of Evolving Phase Boundaries in the Plane, Oxford Press, 1993

[G3] M. E. Gurtin, The nature of configurational forces, Arch. Rational Mech. Anal. 131, 67-100 (1995)

[Mu] W. W. Mullins, Two-dimensional motion of idealized grain boundaries, J. Appl. Phys. 27, 900$904(1956)$ 